\title{
Random Variables and Product of Probability Spaces ${ }^{1}$
}

\author{
Hiroyuki Okazaki \\ Shinshu University \\ Nagano, Japan
}

\author{
Yasunari Shidama \\ Shinshu University \\ Nagano, Japan
}

Summary. We have been working on the formalization of the probability and the randomness. In 15 and 16, we formalized some theorems concerning the real-valued random variables and the product of two probability spaces. In this article, we present the generalized formalization of [15] and [16]. First, we formalize the random variables of arbitrary set and prove the equivalence between random variable on $\Sigma$, Borel sets and a real-valued random variable on $\Sigma$. Next, we formalize the product of countably infinite probability spaces.

MML identifier: RANDOM_3, version: 8.1.01 5.7.1169

The notation and terminology used in this paper have been introduced in the following articles: [1], [14], [12], 44, [11], [18, [7], [8], [5], 2], 3], [9], [13, [22], [15], [16], 20], 21], [17, [19], 6], and [10].

\section{RANDOM VARIABLES}

In this paper $\Omega, \Omega_{1}, \Omega_{2}$ denote non empty sets, $\Sigma$ denotes a $\sigma$-field of subsets of $\Omega, S_{1}$ denotes a $\sigma$-field of subsets of $\Omega_{1}$, and $S_{2}$ denotes a $\sigma$-field of subsets of $\Omega_{2}$.

Now we state the proposition:

(1) Let us consider a non empty set $B$ and a function $f$. Then $f^{-1}(\bigcup B)=$ $\bigcup\left\{f^{-1}(Y)\right.$ where $Y$ is an element of $B$ : not contradiction $\}$.

Let us consider a function $f$ from $\Omega_{1}$ into $\Omega_{2}$, a sequence $B$ of subsets of $\Omega_{2}$, and a sequence $D$ of subsets of $\Omega_{1}$. Now we state the propositions:

\footnotetext{
${ }^{1}$ The 1st author was supported by JSPS KAKENHI 21240001, and the 2nd author was supported by JSPS KAKENHI 22300285. 
(2) If for every element $n$ of $\mathbb{N}, D(n)=f^{-1}(B(n))$, then $f^{-1}(\bigcup B)=\bigcup D$.

(3) If for every element $n$ of $\mathbb{N}, D(n)=f^{-1}(B(n))$, then $f^{-1}($ Intersection $B)=$ Intersection $D$.

Now we state the propositions:

(4) Let us consider a function $F$ from $\Omega$ into $\mathbb{R}$ and a real number $r$. Suppose $F$ is a real-valued random variable on $\Sigma$. Then $F^{-1}(]-\infty, r[) \in \Sigma$. Proof: Consider $X$ being an element of $\Sigma$ such that $X=\Omega$ and $F$ is measurable on $X$. For every element $z, z \in F^{-1}(]-\infty, r[)$ iff $z \in \Omega_{\Sigma} \cap \operatorname{LE}-\operatorname{dom}(F, r)$.

(5) Let us consider a function $F$ from $\Omega$ into $\mathbb{R}$. Suppose $F$ is a real-valued random variable on $\Sigma$. Then $\{x$ where $x$ is an element of the Borel sets : $F^{-1}(x)$ is element of $\left.\Sigma\right\}$ is a $\sigma$-field of subsets of $\mathbb{R}$. The theorem is a consequence of (4) and (3). Proof: Set $S=\{x$ where $x$ is an element of the Borel sets : $F^{-1}(x)$ is an element of $\left.\Sigma\right\}$. For every element $x$ such that $x \in S$ holds $x \in$ the Borel sets. Set $r_{0}=$ the element of $\mathbb{R}$. Reconsider $y_{0}=$ halfline $\left(r_{0}\right)$ as an element of the Borel sets. For every subset $A$ of $\mathbb{R}$ such that $A \in S$ holds $A^{\mathrm{c}} \in S$. For every sequence $A_{1}$ of subsets of $\mathbb{R}$ such that $\operatorname{rng} A_{1} \subseteq S$ holds Intersection $A_{1} \in S$.

Let us consider a function $f$ from $\Omega$ into $\mathbb{R}$. Now we state the propositions:

(6) Suppose $f$ is a real-valued random variable on $\Sigma$. Then $\{x$ where $x$ is an element of the Borel sets : $f^{-1}(x)$ is an element of $\left.\Sigma\right\}=$ the Borel sets.

(7) $f$ is random variable on $\Sigma$ and the Borel sets if and only if $f$ is a realvalued random variable on $\Sigma$.

(8) The set of random variables on $\Sigma$ and the Borel sets $=$ the real-valued random variables set on $\Sigma$.

Let us consider $\Omega_{1}, \Omega_{2}, S_{1}$, and $S_{2}$. Let $F$ be a function from $\Omega_{1}$ into $\Omega_{2}$. We say that $F$ is $\left(S_{1}, S_{2}\right)$-random variable-like if and only if

(Def. 1) $\quad F$ is random variable on $S_{1}$ and $S_{2}$.

Observe that there exists a function from $\Omega_{1}$ into $\Omega_{2}$ which is $\left(S_{1}, S_{2}\right)$ random variable-like.

A random variable of $S_{1}$ and $S_{2}$ is an $\left(S_{1}, S_{2}\right)$-random variable-like function from $\Omega_{1}$ into $\Omega_{2}$. Now we state the proposition:

(9) Let us consider a function $f$ from $\Omega$ into $\mathbb{R}$. Then $f$ is a random variable of $\Sigma$ and the Borel sets if and only if $f$ is a real-valued random variable on $\Sigma$.

Let $F$ be a function. We say that $F$ is random variable family-like if and only if

(Def. 2) Let us consider a set $x$. Suppose $x \in \operatorname{dom} F$. Then there exist non empty sets $\Omega_{1}, \Omega_{2}$ and there exists a $\sigma$-field $S_{1}$ of subsets of $\Omega_{1}$ and there exists 
a $\sigma$-field $S_{2}$ of subsets of $\Omega_{2}$ and there exists a random variable $f$ of $S_{1}$ and $S_{2}$ such that $F(x)=f$.

One can verify that there exists a function which is random variable familylike.

A random variable family is a random variable family-like function. In this paper $F$ denotes a random variable of $S_{1}$ and $S_{2}$.

Let $Y$ be a non empty set, $S$ be a $\sigma$-field of subsets of $Y$, and $F$ be a function. We say that $F$ is $S$-measure valued if and only if

(Def. 3) Let us consider a set $x$. If $x \in \operatorname{dom} F$, then there exists a $\sigma$-measure $M$ on $S$ such that $F(x)=M$.

Note that there exists a function which is $S$-measure valued.

Let $F$ be a function. We say that $F$ is $S$-probability valued if and only if

(Def. 4) Let us consider a set $x$. If $x \in \operatorname{dom} F$, then there exists a probability $P$ on $S$ such that $F(x)=P$.

Let us note that there exists a function which is $S$-probability valued.

Let $X, Y$ be non empty sets. One can verify that there exists an $S$-probability valued function which is $X$-defined.

One can verify that there exists an $X$-defined $S$-probability valued function which is total.

Let $Y$ be a non empty set. Let us note that every function which is $S$ probability valued is also $S$-measure valued.

if

Let $F$ be a function. We say that $F$ is $S$-random variable family if and only

(Def. 5) Let us consider a set $x$. Suppose $x \in \operatorname{dom} F$. Then there exists a realvalued random variable $Z$ on $S$ such that $F(x)=Z$.

Observe that there exists a function which is $S$-random variable family.

Now we state the propositions:

(10) Let us consider an element $y$ of $S_{2}$. Suppose $y \neq \emptyset$. Then $\{z$ where $z$ is an element of $\Omega_{1}: F(z)$ is an element of $\left.y\right\}=F^{-1}(y)$. Proof: Set $D=\left\{z\right.$ where $z$ is an element of $\Omega_{1}: F(z)$ is an element of $\left.y\right\}$. For every element $x, x \in D$ iff $x \in F^{-1}(y)$.

(11) Let us consider a random variable $F$ of $S_{1}$ and $S_{2}$. Then

(i) $\left\{x\right.$ where $x$ is a subset of $\Omega_{1}$ : there exists an element $y$ of $S_{2}$ such that $\left.x=F^{-1}(y)\right\} \subseteq S_{1}$, and

(ii) $\left\{x\right.$ where $x$ is a subset of $\Omega_{1}$ : there exists an element $y$ of $S_{2}$ such that $\left.x=F^{-1}(y)\right\}$ is a $\sigma$-field of subsets of $\Omega_{1}$.

The theorem is a consequence of (3). Proof: Set $S=\{x$ where $x$ is a subset of $\Omega_{1}$ : there exists an element $y$ of $S_{2}$ such that $\left.x=F^{-1}(y)\right\}$. For every element $x$ such that $x \in S$ holds $x \in S_{1}$. For every subset $A$ of 
$\Omega_{1}$ such that $A \in S$ holds $A^{\mathrm{c}} \in S$. For every sequence $A_{1}$ of subsets of $\Omega_{1}$ such that $\operatorname{rng} A_{1} \subseteq S$ holds Intersection $A_{1} \in S$.

Let us consider $\Omega_{1}, \Omega_{2}, S_{1}$, and $S_{2}$. Let $M$ be a measure on $S_{1}$ and $F$ be a random variable of $S_{1}$ and $S_{2}$. The functor the image measure of $F$ and $M$ yielding a measure on $S_{2}$ is defined by

(Def. 6) Let us consider an element $y$ of $S_{2}$. Then it $(y)=M\left(F^{-1}(y)\right)$.

Let $M$ be a $\sigma$-measure on $S_{1}$. Note that the image measure of $F$ and $M$ is $\sigma$-additive.

Now we state the proposition:

(12) Let us consider a probability $P$ on $S_{1}$ and a random variable $F$ of $S_{1}$ and $S_{2}$. Then (the image measure of $F$ and P2M $\left.P\right)\left(\Omega_{2}\right)=1$.

Let us consider $\Omega_{1}, \Omega_{2}, S_{1}$, and $S_{2}$. Let $P$ be a probability on $S_{1}$ and $F$ be a random variable of $S_{1}$ and $S_{2}$. The functor probability $(F, P)$ yielding a probability on $S_{2}$ is defined by the term

(Def. 7) M2P the image measure of $F$ and P2M $P$.

Now we state the propositions:

(13) Let us consider a probability $P$ on $S_{1}$ and a random variable $F$ of $S_{1}$ and $S_{2}$. Then probability $(F, P)=$ the image measure of $F$ and P2M $P$. The theorem is a consequence of (12).

(14) Let us consider a probability $P$ on $S_{1}$, a random variable $F$ of $S_{1}$ and $S_{2}$, and a set $y$. If $y \in S_{2}$, then $(\operatorname{probability}(F, P))(y)=P\left(F^{-1}(y)\right)$. The theorem is a consequence of (13).

(15) Every function from $\Omega_{1}$ into $\Omega_{2}$ is a random variable of the trivial $\sigma$-field of $\Omega_{1}$ and the trivial $\sigma$-field of $\Omega_{2}$.

(16) Let us consider a non empty set $S$. Then every non empty finite sequence of elements of $S$ is a random variable of the trivial $\sigma$-field of Seg len $F$ and the trivial $\sigma$-field of $S$. The theorem is a consequence of (15).

(17) Let us consider finite non empty sets $V, S$, a random variable $G$ of the trivial $\sigma$-field of $V$ and the trivial $\sigma$-field of $S$, and a set $y$. Suppose $y \in$ the trivial $\sigma$-field of $S$. Then (probability $(G$, the trivial probability of $V))(y)=\frac{\overline{\overline{G^{-1}(y)}}}{\overline{\bar{V}}}$. The theorem is a consequence of $(14)$.

(18) Let us consider a finite non empty set $S$, a non empty finite sequence $s$ of elements of $S$, and a set $x$. Suppose $x \in S$. Then there exists a random variable $G$ of the trivial $\sigma$-field of Seg len $s$ and the trivial $\sigma$-field of $S$ such that

(i) $G=s$, and

(ii) $(\operatorname{probability}(G$, the trivial probability of Seg len $s))(\{x\})=\operatorname{Prob}_{\mathrm{D}}(x, s)$. The theorem is a consequence of (16) and (17). 


\section{Product of Probability Spaces}

Let $D$ be a non-empty many sorted set indexed by $\mathbb{N}$ and $n$ be a natural number. One can check that $D(n)$ is non empty.

Let $S, F$ be many sorted sets indexed by $\mathbb{N}$. We say that $F$ is $\sigma$-field $S$ sequence-like if and only if

(Def. 8) Let us consider a natural number $n$. Then $F(n)$ is a $\sigma$-field of subsets of $S(n)$.

Let $S$ be a many sorted set indexed by $\mathbb{N}$. Let us observe that there exists a many sorted set indexed by $\mathbb{N}$ which is $\sigma$-field $S$-sequence-like.

Let $D$ be a many sorted set indexed by $\mathbb{N}$. A $\sigma$-field sequence of $D$ is a $\sigma$-field $D$-sequence-like many sorted set indexed by $\mathbb{N}$. Let $S$ be a $\sigma$-field sequence of $D$ and $n$ be a natural number. Note that the functor $S(n)$ yields a $\sigma$-field of subsets of $D(n)$. Let $D$ be a non-empty many sorted set indexed by $\mathbb{N}$. Let $M$ be a many sorted set indexed by $\mathbb{N}$. We say that $M$ is $S$-probability sequence-like if and only if

(Def. 9) Let us consider a natural number $n$. Then $M(n)$ is a probability on $S(n)$.

Observe that there exists a many sorted set indexed by $\mathbb{N}$ which is $S$ probability sequence-like.

A probability sequence of $S$ is an $S$-probability sequence-like many sorted set indexed by $\mathbb{N}$. Let $P$ be a probability sequence of $S$ and $n$ be a natural number. One can verify that the functor $P(n)$ yields a probability on $S(n)$. Let $D$ be a many sorted set indexed by $\mathbb{N}$. The functor the product domain $D$ yielding a many sorted set indexed by $\mathbb{N}$ is defined by

(Def. 10) (i) $i t(0)=D(0)$, and

(ii) for every natural number $i, i t(i+1)=i t(i) \times D(i+1)$.

Now we state the proposition:

(19) Let us consider a many sorted set $D$ indexed by $\mathbb{N}$. Then

(i) (the product domain $D)(0)=D(0)$, and

(ii) (the product domain $D)(1)=D(0) \times D(1)$, and

(iii) (the product domain $D)(2)=D(0) \times D(1) \times D(2)$, and

(iv) (the product domain $D)(3)=D(0) \times D(1) \times D(2) \times D(3)$.

Let $D$ be a non-empty many sorted set indexed by $\mathbb{N}$. Let us note that the product domain $D$ is non-empty.

Let $D$ be a finite-yielding many sorted set indexed by $\mathbb{N}$. One can check that the product domain $D$ is finite-yielding.

Let us consider $\Omega$ and $\Sigma$. Let $P$ be a set. Assume $P$ is a probability on $\Sigma$. The functor modetrans $(P, \Sigma)$ yielding a probability on $\Sigma$ is defined by the term (Def. 11) $P$. 
Let $D$ be a finite-yielding non-empty many sorted set indexed by $\mathbb{N}$. The functor the trivial $\sigma$-field sequence $D$ yielding a $\sigma$-field sequence of $D$ is defined by

(Def. 12) Let us consider a natural number $n$. Then $i t(n)=$ the trivial $\sigma$-field of $D(n)$.

Let $P$ be a probability sequence of the trivial $\sigma$-field sequence $D$ and $n$ be a natural number. One can check that the functor $P(n)$ yields a probability on the trivial $\sigma$-field of $D(n)$. The functor ProductProbability $(P, D)$ yielding a many sorted set indexed by $\mathbb{N}$ is defined by

(Def. 13) (i) it $(0)=P(0)$, and

(ii) for every natural number $i, i t(i+1)=$

Product-Probability ( (the product domain $D)(i), D(i+1)$, modetrans (it $(i)$, the trivial $\sigma$-field of (the product domain $D)(i)$ ), $P(i+1)$ ).

Let us consider a finite-yielding non-empty many sorted set $D$ indexed by $\mathbb{N}$, a probability sequence $P$ of the trivial $\sigma$-field sequence $D$, and a natural number $n$. Now we state the propositions:

(20) (ProductProbability $(P, D))(n)$ is a probability on the trivial $\sigma$-field of (the product domain $D)(n)$.

(21) There exists a probability $P_{4}$ on the trivial $\sigma$-field of (the product domain $D)(n)$ such that

(i) $P_{4}=(\operatorname{ProductProbability}(P, D))(n)$, and

(ii) (ProductProbability $(P, D))(n+1)=$ Product-Probability $(($ the product domain $\left.D)(n), D(n+1), P_{4}, P(n+1)\right)$.

Now we state the proposition:

(22) Let us consider a finite-yielding non-empty many sorted set $D$ indexed by $\mathbb{N}$ and a probability sequence $P$ of the trivial $\sigma$-field sequence $D$. Then

(i) $(\operatorname{ProductProbability}(P, D))(0)=P(0)$, and

(ii) $(\operatorname{ProductProbability}(P, D))(1)=$

Product-Probability $(D(0), D(1), P(0), P(1))$, and

(iii) there exists a probability $P_{1}$ on the trivial $\sigma$-field of $D(0) \times D(1)$ such that $P_{1}=(\operatorname{ProductProbability}(P, D))(1)$ and (ProductProbability $(P$, $D))(2)=$ Product-Probability $\left(D(0) \times D(1), D(2), P_{1}, P(2)\right)$, and

(iv) there exists a probability $P_{2}$ on the trivial $\sigma$-field of $D(0) \times D(1) \times$ $D(2)$ such that $P_{2}=(\operatorname{ProductProbability}(P, D))(2)$ and $(\operatorname{ProductProbability}(P, D))(3)=\operatorname{Product}-\operatorname{Probability}(D(0) \times D(1) \times$ $\left.D(2), D(3), P_{2}, P(3)\right)$, and

(v) there exists a probability $P_{3}$ on the trivial $\sigma$-field of $D(0) \times D(1) \times$ $D(2) \times D(3)$ such that $P_{3}=(\operatorname{ProductProbability}(P, D))(3)$ and 


$$
\begin{aligned}
& \text { ProductProbability }(P, D))(4)=\text { Product-Probability }(D(0) \times D(1) \times \\
& \left.D(2) \times D(3), D(4), P_{3}, P(4)\right) .
\end{aligned}
$$

The theorem is a consequence of (19) and (21).

\section{REFERENCES}

[1] Grzegorz Bancerek. Cardinal numbers. Formalized Mathematics, 1(2):377-382, 1990.

[2] Grzegorz Bancerek. The fundamental properties of natural numbers. Formalized Mathematics, 1(1):41-46, 1990.

[3] Grzegorz Bancerek. The ordinal numbers. Formalized Mathematics, 1(1):91-96, 1990.

[4] Grzegorz Bancerek and Krzysztof Hryniewiecki. Segments of natural numbers and finite sequences. Formalized Mathematics, 1(1):107-114, 1990.

[5] Józef Białas. The $\sigma$-additive measure theory. Formalized Mathematics, 2(2):263-270, 1991.

[6] Józef Białas. Series of positive real numbers. Measure theory. Formalized Mathematics, 2(1):173-183, 1991.

[7] Czesław Byliński. Functions and their basic properties. Formalized Mathematics, 1(1): 55-65, 1990.

[8] Czesław Byliński. Functions from a set to a set. Formalized Mathematics, 1(1):153-164, 1990.

[9] Czesław Byliński. Partial functions. Formalized Mathematics, 1(2):357-367, 1990.

[10] Czesław Byliński. Some basic properties of sets. Formalized Mathematics, 1(1):47-53, 1990.

[11] Agata Darmochwał. Finite sets. Formalized Mathematics, 1(1):165-167, 1990.

[12] Peter Jaeger. Elementary introduction to stochastic finance in discrete time. Formalized Mathematics, 20(1):1-5, 2012. doi:10.2478/v10037-012-0001-5

[13] Andrzej Nędzusiak. $\sigma$-fields and probability. Formalized Mathematics, 1(2):401-407, 1990.

[14] Hiroyuki Okazaki. Probability on finite and discrete set and uniform distribution. Formalized Mathematics, 17(2):173-178, 2009. doi 10.2478/v10037-009-0020-z

[15] Hiroyuki Okazaki and Yasunari Shidama. Probability on finite set and real-valued random variables. Formalized Mathematics, 17(2):129-136, 2009. doi 10.2478/v10037-009-0014-x

[16] Hiroyuki Okazaki and Yasunari Shidama. Probability measure on discrete spaces and algebra of real-valued random variables. Formalized Mathematics, 18(4):213-217, 2010. doi $10.2478 /$ v10037-010-0026-6

[17] Beata Padlewska. Families of sets. Formalized Mathematics, 1(1):147-152, 1990.

[18] Andrzej Trybulec and Agata Darmochwał. Boolean domains. Formalized Mathematics, 1 (1):187-190, 1990.

[19] Zinaida Trybulec. Properties of subsets. Formalized Mathematics, 1(1):67-71, 1990.

[20] Edmund Woronowicz. Relations and their basic properties. Formalized Mathematics, 1 (1):73-83, 1990.

[21] Edmund Woronowicz. Relations defined on sets. Formalized Mathematics, 1(1):181-186, 1990.

[22] Bo Zhang, Hiroshi Yamazaki, and Yatsuka Nakamura. The relevance of measure and probability, and definition of completeness of probability. Formalized Mathematics, 14 (4):225-229, 2006. doi $10.2478 / \mathrm{v} 10037-006-0026-8$.

Received December 1, 2012 